
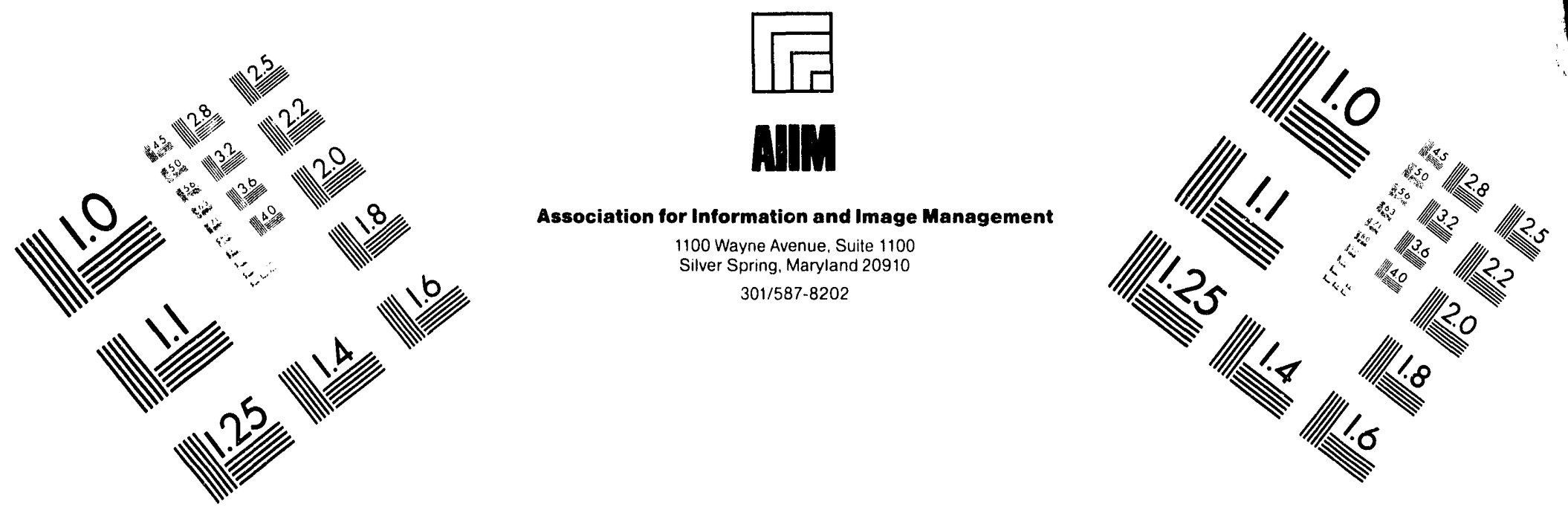

Centimeter

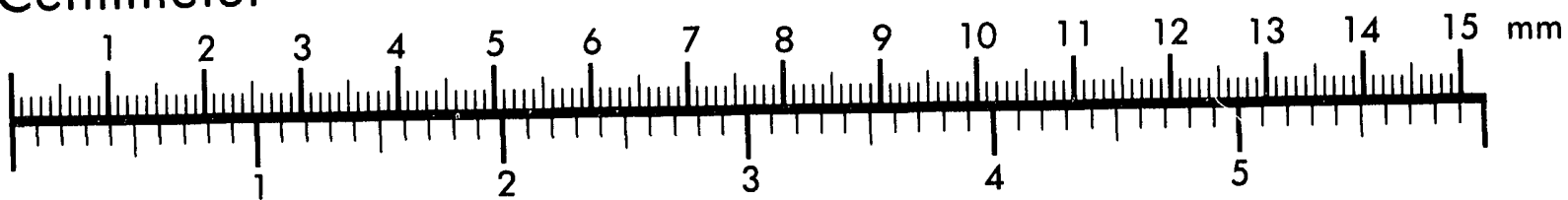
Inches
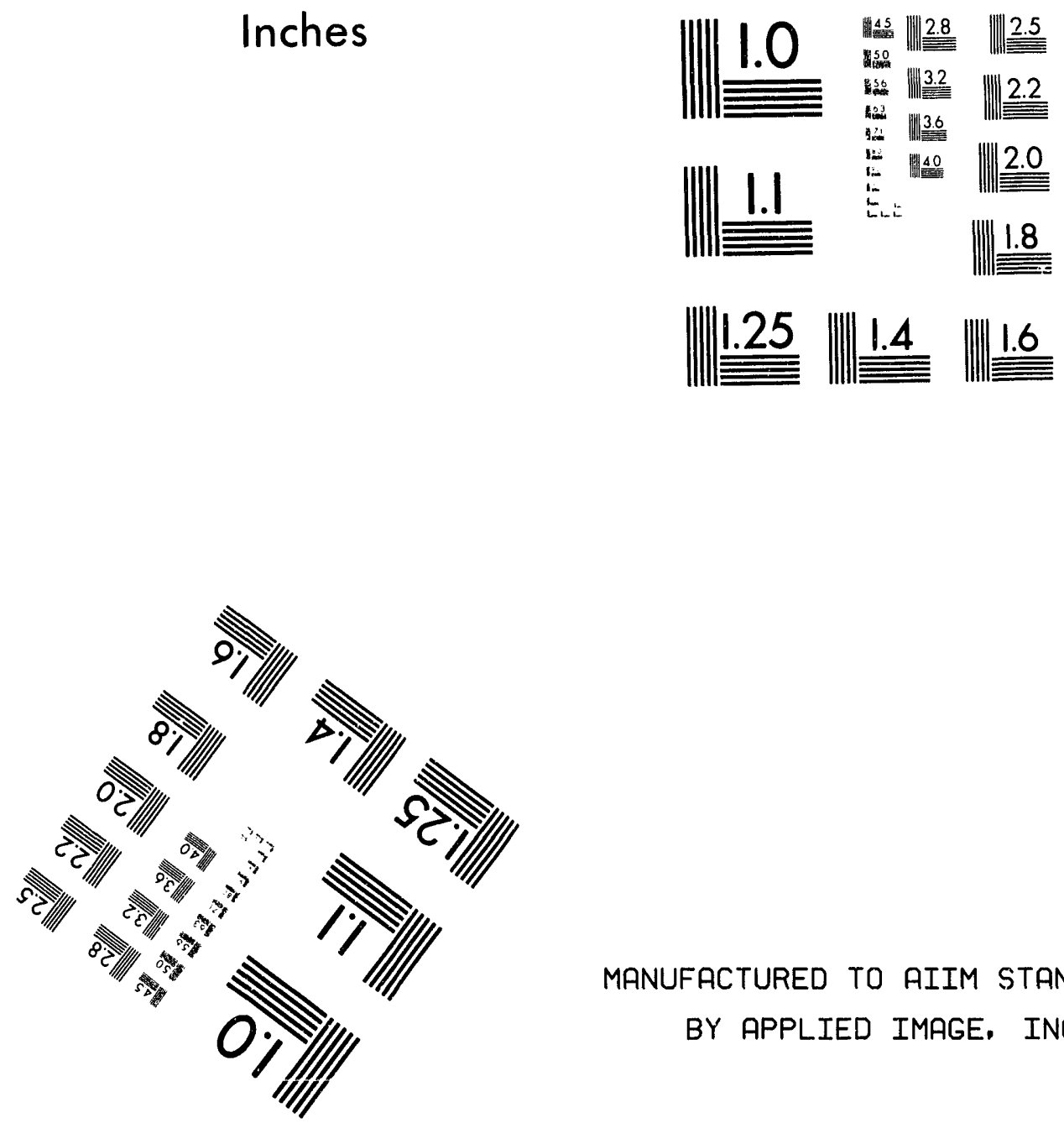

MANUFACTURED TO AIIM STANDARDS

$$
\text { BY APPLIED IMAGE, INC. }
$$

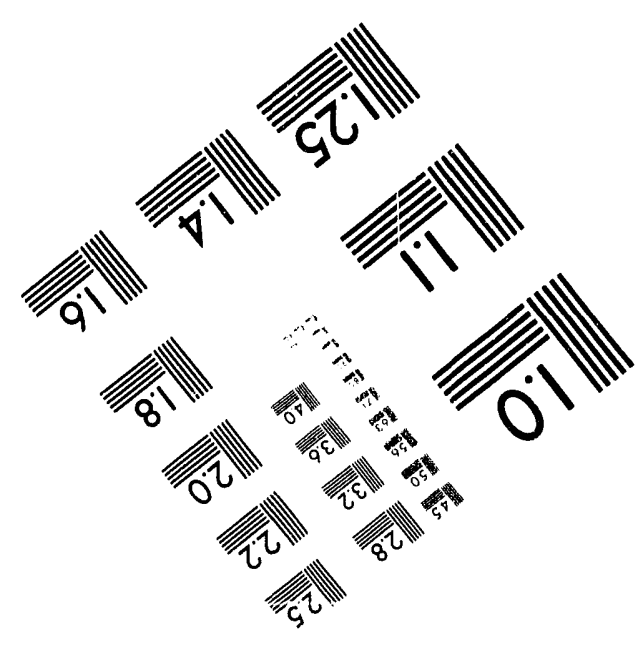



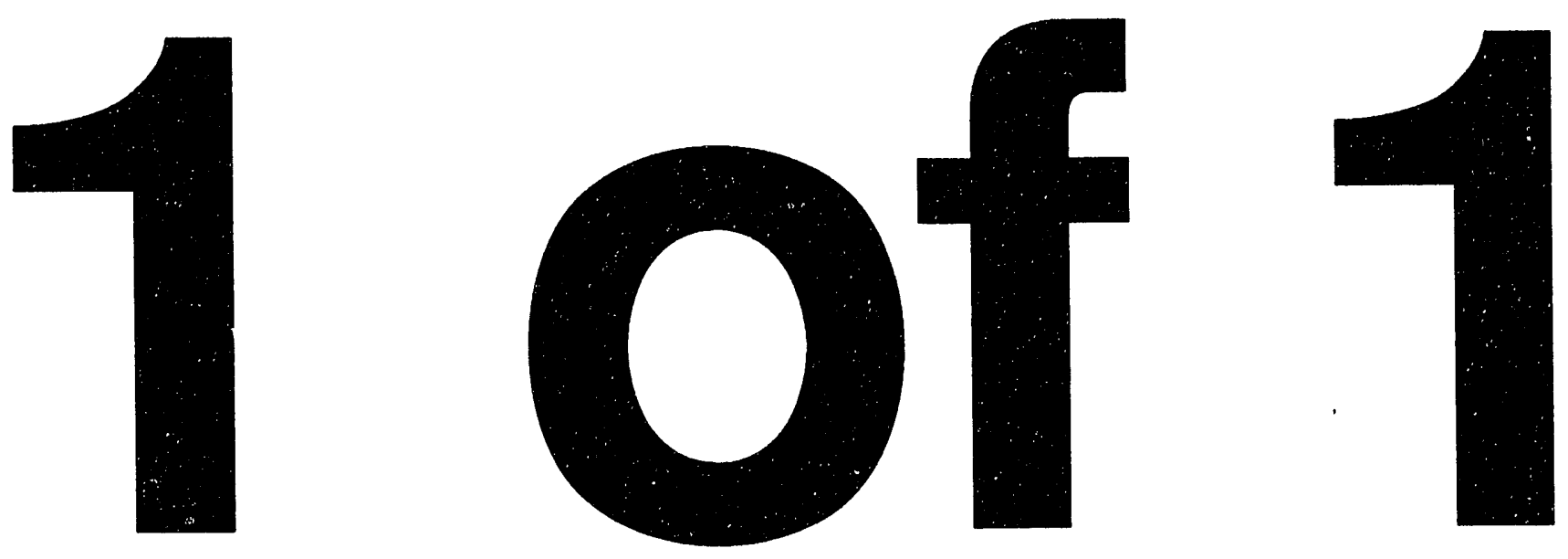
This report was prepared as an account of work spcnsored by an agency of the United States Government. Neither the United States Government nor any agency thereof, nor any of their SLAC/SSRL-0010 employees, makes any warranty, express or implied, or assumes any legal liability or responsibility for the accuracy, completeness, or usefulness of any information, apparatus, product, or process disclosed, or represents that its use would not infringe privately owned rights. Reference herein to any specific commercial product, process, or service by trade name, trademark, manufacturer, or otherwise does not necessarily constitute or imply its endorsement, recommendation, or favoring by the United States Government or any agency thereof. The views and opinions of authors expressed herein do not necessarily state or reflect those of the United States Government or any agency thereof.

\title{
ANISOTROPIC PHASE SEPARATION IN AMORPHOUS Fe-Ge ALLOYS*
}

\author{
MICHAEL J. REGAN and ARTHUR BIENENSTOCK
}

\author{
Stanford Linear Accelerator Center \\ Stanford Synchrotron Radiation Laboratory \\ Stanford University, Stanford, CA 94309
}

\begin{abstract}
Magnetron sputtered amorphous $\mathrm{Fe}_{\mathrm{x}} \mathrm{Ge}_{100-\mathrm{x}}$ films have been examined with anomalous small-angle $x$-ray scattering (ASAXS) in an attempt to characterize composition fluctuations which have been previously reported in this system. Films grown under various deposition conditions have been studied, with the scattering vector both in and oblique to the plane of the films, to search for anisotropy. By manipulating the deposited power flux and rates of growth, films which have the same composition can be grown to different states of phase separation. The total correlation functions have been calculated from the oblique scattering experiments. The anisotropy can be successfully modeled as a close-packing of oriented prolate ellipsoidal particles, with the elongated axis along the direction of film growth. A method for using these measurements to determine the compositions of the phase-separating species has been developed and utilized. The results indicate phase separation into $\mathrm{a}-\mathrm{Ge}$ and $\mathrm{a}-\mathrm{FeGe}_{2}$ for the $\mathrm{a}-\mathrm{Fe}_{\mathbf{x}} \mathrm{Ge}_{100-\mathrm{x}}(\mathrm{x}<33)$ alloy.
\end{abstract}

Presented at the 1993 Spring Meeting of the Materials Research Society, San Francisco, CA, April 12-16, 1993.

*Work supported by the US Department of Energy under contract DE-AC03-76SF00515

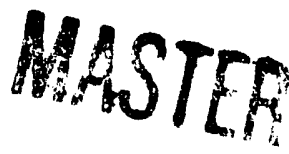




\section{INTRODUCTION}

A common approach to understanding vapor-deposited amorphous alloys is to focus on the short-range order, while assuming chemical homogeneity and isotropy. An interesting problem arises, however, when the equilibrium phase diagram indicates that there is no solid solubility throughout the composition range of interest. It is not obvious that the quasi-equilibrium state of the amorphous alloy can be treated as homogeneous and not phase separated. This is a fundamental problem, since most phase transitions (e.g. metal/insulator, para/ferromagnet) in amorphous materials assume little if any order exists beyond the first few neighbors.

Past experiments of Rice et al..$^{1}$ and Lorentz et al. ${ }^{2}$ show that composition fluctuations of some kind exist throughout most of the composition range $(0<x<75)$ of triodesputtered a- $\mathrm{Fe}_{\mathrm{x}} \mathrm{Ge}_{100-\mathrm{x}}$ films. The phase diagram of $\mathrm{Fe}_{\mathrm{x}} \mathrm{Ge}_{100-\mathrm{x}}$ shows no solid solubility throughout most of the composition range, particularly for $\mathrm{x}<33$ where the equilibrium state is a mixture of $\mathrm{c}-\mathrm{Ge}$ and $\mathrm{c}-\mathrm{FeGe}_{2}$. Among its more interesting properties, the alloy exhibits a semiconductor to metal transition near 10-15 atomic percent $\mathrm{Fe}$. We have focused on better understanding the apparent phase separation in the composition range in which this transition occurs $(x<33)$. By varying growth parameters and checking for anisotropy, we have been able to show that deposition conditions critically affect amorphous phase growth and that the phases are not packed isotropically but rather depend quite significantly on the film surface.

\section{EXPERIMENT}

a- $\mathrm{Fe}_{\mathrm{x}} \mathrm{Ge}_{100-\mathrm{x}}(\mathrm{x}<33)$ films were prepared by magnetron co-sputtering of elemental targets onto Si wafers attached to a rapidly rotating substrate table. The Si substrates were then removed and films ( $\sim 5-8$ microns thick) rendered free-standing after immersion in a warm $\mathrm{KOH}$ bath. ${ }^{3}$ Samples that are sufficiently thick and free-standing are $x=6,11,14$, and 18; those that are not free-standing but are kapton-supported, $x=0$, 26,44 , and 47 . Initial characterization of the samples included a check for crystallization with a conventional Picker diffractometer, a rough determination of the composition with an electron microprobe and a thickness measurement with an alpha-step profilometer. 
More accurate values of the composition have been obtained using x-ray absorption methods. ${ }^{4}$

The ASAXS experiment was performed on the eight-pole focused wiggler end-station 4-2 at the Stanford Synchrotron Radiation Laboratory. The basic line consists of a bent cylindrical mirror and a double-crystal monochromator. For this experiment, we used $\mathrm{Si}(111)$ crystals and worked at five incident $\mathrm{x}$-ray energies below the $\mathrm{Fe} \mathrm{K}$ edge $(-200$, $-100,-50,-20,-10 \mathrm{eV}$, edge at $7112 \mathrm{eV}$ ) and the Ge K edge (edge at $11103 \mathrm{eV}$ ). In order to test for anisotropy, oblique transmission measurements 5 which allow for various orientations of the scattering vector were performed. By tilting the sample to angle $\omega$, the scattering vector rotates out of the surface plane, and correlations in electron density are projected onto the plane defined at angle $\omega$ with respect to the surface plane $(\omega=0)$--see figure 1. The data were background subtracted, scaled per irradiated volume and absorption, and placed on an absolute scale ( $10 \%$ error) with a lupolen secondary standard. ${ }^{6}$ We report results in terms of the structure factor, $S(\vec{k})$, which is the fourier transform of the electron density pair correlation function, $\gamma(\vec{r})$. The total cross-sections (integrated intensities) have been obtained by integrating over both the magnitude of the scattering vector as well as the polar angle $\omega$. The details of the data collection, normalization, and manipulation procedures will be summarized more completely by Regan. $^{7}$

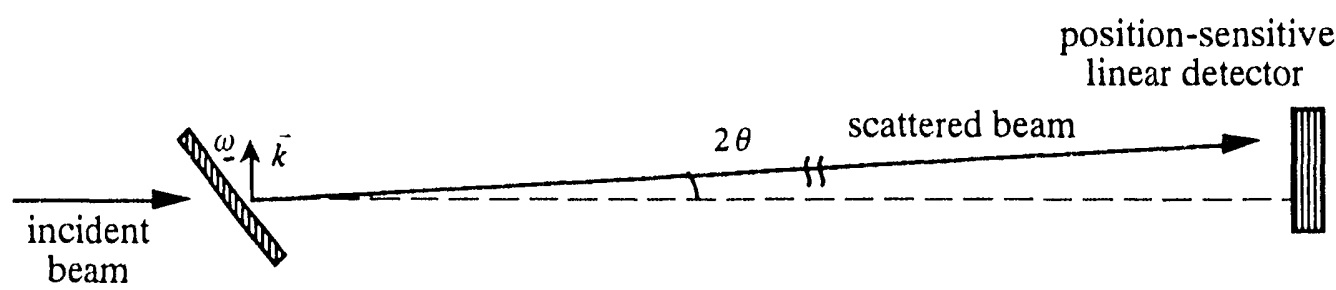

Figure 1. Scattering geometry for oblique transmission experiment.

\section{RESULTS}

Figure 2 illustrates the structure factor $S(k, \omega)$ determined from the oblique transmission experiment for a- $\mathrm{Fe}_{6} \mathrm{Ge}_{94}$ at $6912 \mathrm{eV}$. A strong anisotropy appears, observable from the change in the scattering peak with sample orientation. As the sample is tilted, the peak shifts inward to smaller $k$, monotonically decreasing with tilt angle. These data indicate that there are in-plane correlations of some kind at approximately $25 \AA$, which become gradually weaker and of increasingly greater distance as the scattering vector shifts in orientation from the sample surface towards the sample normal. The anomalous experiments for all tilt angles show that there is little if any dependence on the scattering near the Ge K-edge but a large dependence at the Fe K-edge. Such a result had been obtained earlier at normal incidence. 1

The other samples in this composition range $(x<33)$ illustrate the same behavior. That is, there is a monotonic decrease in scattering intensity and shift inward in peak position with increasing $\omega$. As $x$ is further increased to $x=44$ and $x=47$, however, the SAXS maximum disappears and no scattering is observed throughout the extended range $k=4 \pi \sin \theta / \lambda=0.012$ to $0.6 \AA^{-1}$. This is in contrast to results reported for triode-sputtered a$\mathrm{Fe}_{\mathrm{x}} \mathrm{Ge}_{100-\mathrm{x}}$ alloys, where scattering is observed throughout most of the composition range $(0<x<75)$.

By changing the deposition conditions, films identical in composition can be grown to different quasi-equilibrium phase-separated states. Figure 3 compares the scattering from samples that vary both in composition and target power. By manipulating the target power, a host of variables in the vapor deposition process are altered (e.g. adatom thermal energy and deposition rate), so that it is not surprising that the samples exhibit different states. However, it is not obvious as to which particular state is preferred. Samples grown at 400 watts show a more "advanced" state of phase separation than those at 100 watts; that is, the scattering peaks at longer "d-spacings", the peaks have greater magnitudes and they have smaller FWHMs. The greatest change in "d-spacing" length 
occurs in a- $\mathrm{Fe}_{6} \mathrm{Ge}_{94}$ while only an intensity and FWHM difference is apparent in a$\mathrm{Fe}_{26} \mathrm{Ge}_{74}$.

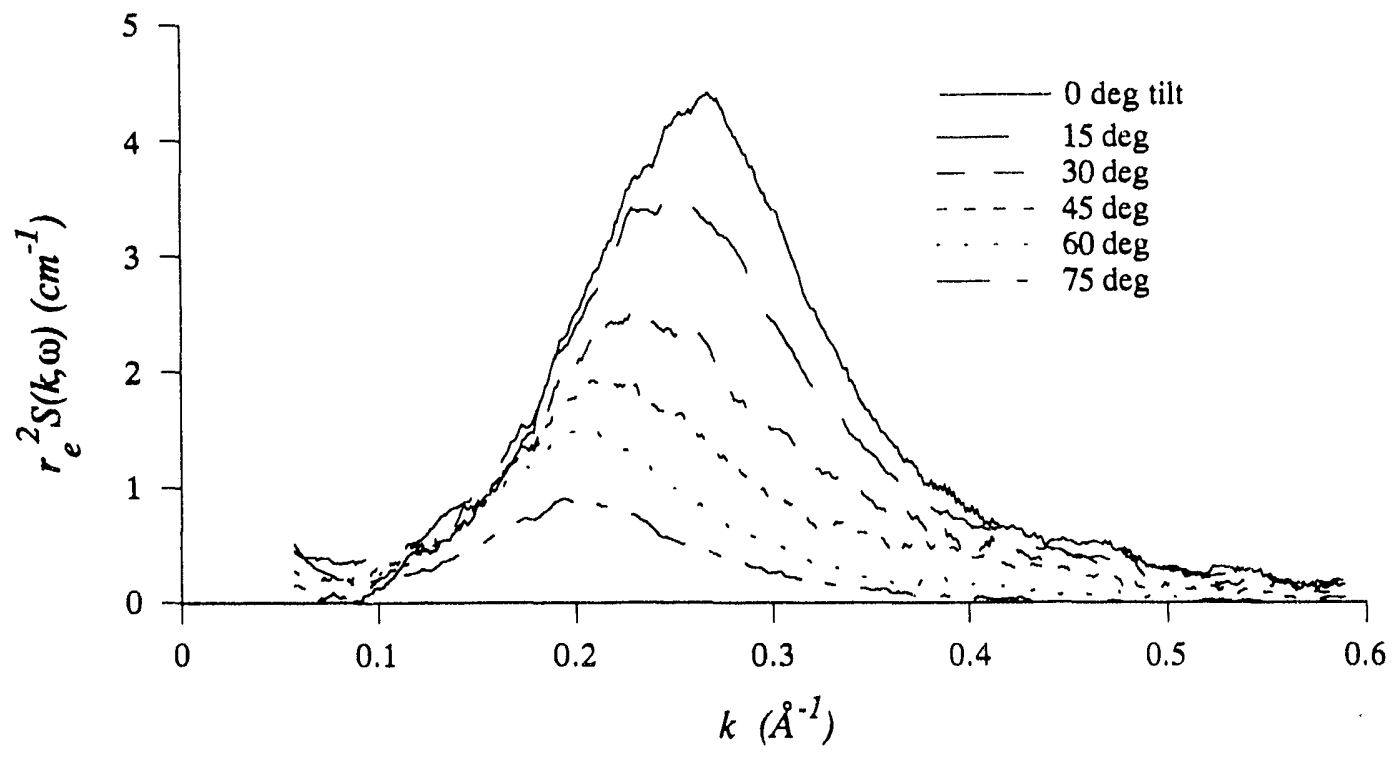

Figure 2. Oblique transmission SAXS experiment. Results are placed on a per volume scale, with $r_{e}$ the classical radius of the electron.

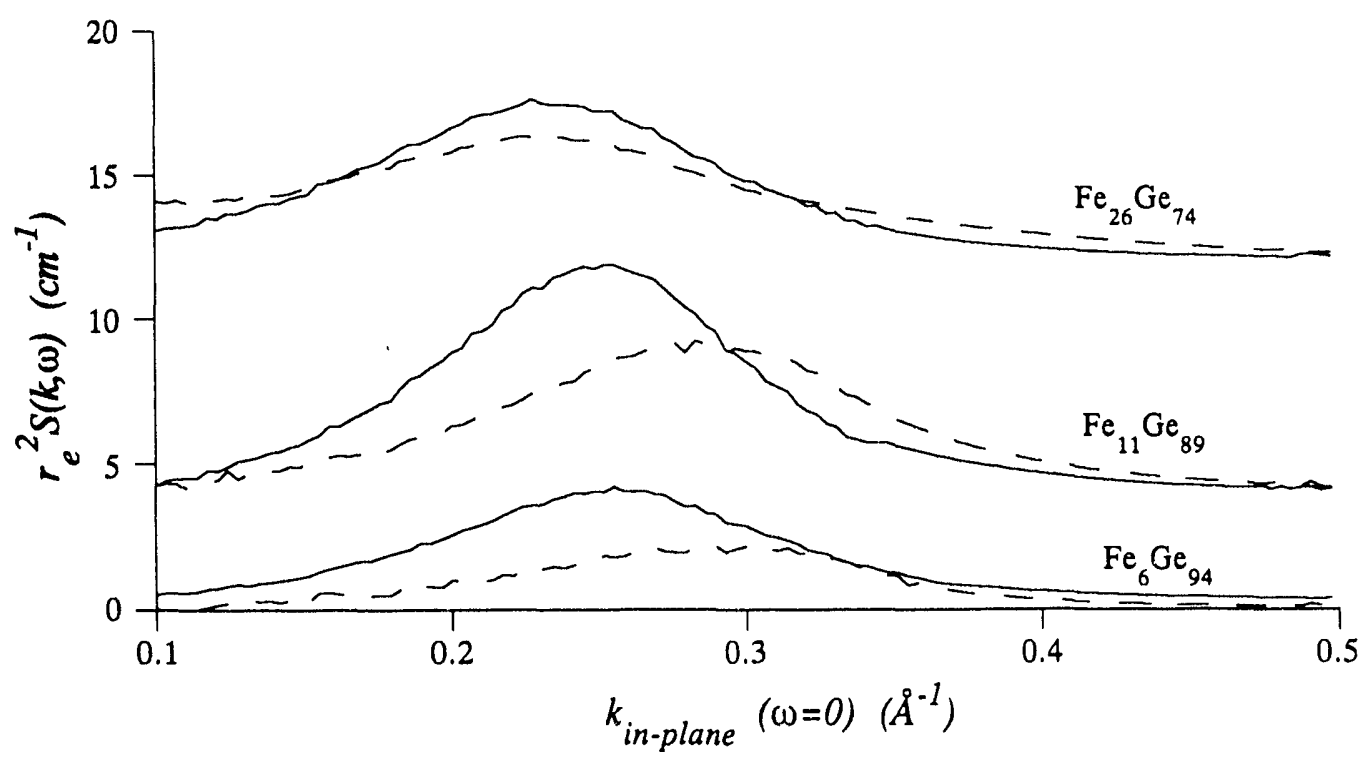

Figure 3. SAXS as a function of composition and power delivered to the sputtering target: solid line, $400 \mathrm{~W}$ power; dashed line 100 W.

\section{MODELS}

The issues that any model must address are not trivial and consequently lead to a deep understanding of the amorphous alloy's structure. The existence of a scattering maximum at a nonzero scattering vector along with the observed anisotropy are difficult to model. Although a unique structure is not discernible from scattering measurements, we have been able to rule out some two-dimensional growth models. Then, more traditional particulate systems are discussed. 


\section{Two-Dimensional Models}

One structure common to vapor-deposited films is that from a columnar growth process. For a culumnar structure, perfect phase correlation exists in the direction of growth $z$; that is, the electron density at $\vec{r}-(x, y, z), \rho(\vec{r})$ (with respect to an arbitrary origin), is identical to the density at point $\vec{r}=(x, y, 0)$. The fourier transform of the pair correlation for a columnar model with in-plane isotropy is

$$
S(k, \omega)=S(k, 0) \frac{\sin ^{2}\left(\frac{T}{2} k \sin \omega\right)}{\left(\frac{T}{2} k \sin \omega\right)^{2}},
$$

with $T$ the film thickness or column height and $S(k, \omega=0)$ the structure factor at normal transmission, and $k$ and $\omega$ reciprocal polar coordinates. Given a similar SAXS spectrum for $\omega=0$ as observed for the $\mathrm{Fe}_{\mathrm{x}} \mathrm{Ge}_{100-\mathrm{x}}$ films, the peak shifts outward and decreases markedly in intensity as the scattering vector changes its orientation to align with the sample normal. It is possible to choose a film thickness $T$ for which the peak initially shifts inward, but for larger angles the peak eventually shifts outward, in disagreement with the data.

The converse to this model is one with no correlation in the direction of growth. That is, the average over all pair correlations that are not in the same layer is 0 , as might be observed in a system with large thermal fluctuations between deposited layers. The pair correlation function for such a model is $\gamma(\vec{x})-\left\langle\rho\left(\bar{x}_{0}\right) \rho\left(\vec{x}+\bar{x}_{0}\right)\right\rangle \delta(z)$, with a structure factor $S(k, \omega)=S(k \cos \omega, 0)$. Again, the model spectra shift outward with $\omega$. The data suggest that non-trivial correlations exist in the direction of growth; otherwise, we would observe a shift outward rather than inward with sample tilt. More sophisticated growth simulations that include tapered and/or shadowed growth might be able to explain the oblique transmission results, however.

\section{Particulate Models}

Whether phase separation occurs via a spinodal decomposition or nucleation process, the late-time structures are fairly similar: there exist regions of well-defined electron density. For the Fe-Ge alloy, regions of well-defined electron density are believed to exist since the large- $k$ side of the scattering pattern exhibits the characteristic $1 / k^{4}$ Porod law of smooth interfaces. This is a part of reciprocal space where interparticle interference effects are less important while scattering from surfaces of the regions in space and electron density dominate.

Consequently, one approach is to consider the scattering due to distinct particles of a particular electron density within a background of different density. The origin of the maximum in the structure factor is then due to either inhomogeneities within the particles or to an interparticle interference effect between groups of particles. By placing the data on an absolute scale, we have been able to show that a simple ellipsoidal inhomogeneous particle model that can explain the anisotropy is inadequate--particles would have to be packed so close together in order to observe the experimental magnitude in scattering that interparticle interference effects between neighboring particles would dominate. The simplest model that thus emerges is a collection of oriented homogeneous ellipsoids.

The observed scattering for the $x=11$ sample is well represented by the theoretical scattering pattern for a collection of oriented prolate ellipsoids of semi-axes $\{4,4,10\} \AA$ (figure 4). The interparticle interference function is approximated by the analytic hard core Born-Green structure factor, ellipsoidal symmetry incorporated by an ellipsoidal hard core repulsion potential of semi-axes $\{21,21,30\} \AA$. It should be noted that the typical hard core repulsion model allows one particle (at closest approach) to touch another. We found that the best agreement with data occurred when a large buffer zone is allowed between the closest point of contact between the particles. 


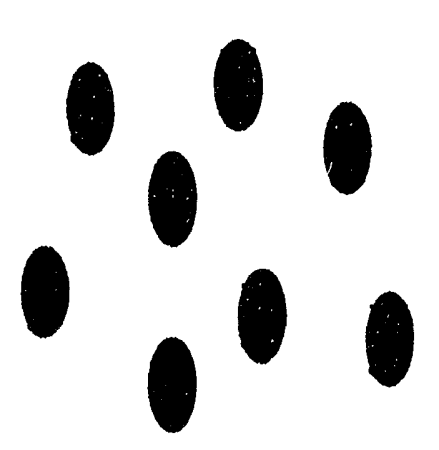

(a)

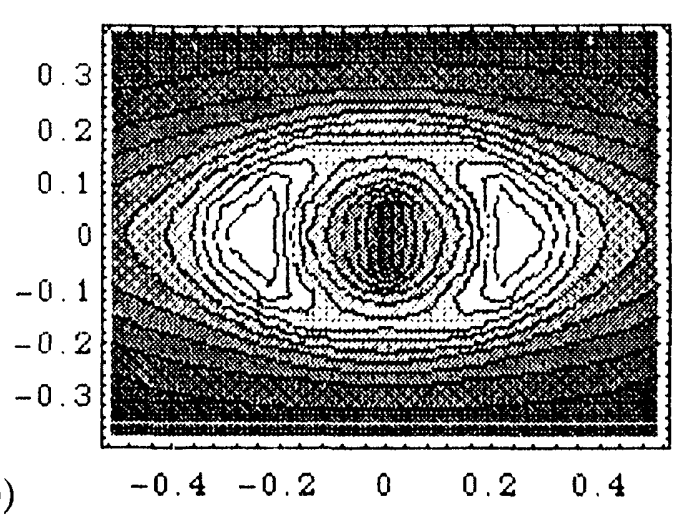

(b)

Figure 4. (a) Configuration and (b) computed structure factor of the oriented prolate ellipsoid model. Horizontal axis: $\omega=0^{\circ}$ (in-plane); vertical axis: $\omega=90^{\circ}$ (direction of growth).

\section{DENSITY CALCULATIONS}

A general, measureable parameter is the mean square fluctuation in electron density $\left\langle\eta^{2}\right\rangle$, proportional to the total cross-section (also known as the integrated intensity). ${ }^{9}\left\langle\eta^{2}\right\rangle$ has been determined by integrating the SAXS spectra over all oblique transmission angles, and we have been able to show that changes in $\left\langle\eta^{2}\right\rangle$ with changes in the anomalous scattering factors at the Fe-edge help determine the composition of the phaseseparating species. Since ASAXS is observed up to but not including $x=0$, it is assumed that one endpoint of phase separation is a-Ge, with the other endpoint obviously in the composition range $x=26$ to $x=44$. For a solid with two distinct phases,

$$
\left\langle\eta^{2}\right\rangle=c(1-c)\left|n_{2}^{F e} f_{F e}(E)+\left(n_{2}^{G e}-n_{1}^{G e}\right) f_{G e}(E)\right|^{2}
$$

with $c$ the volume fraction of material of phase $1, n_{i}^{F e}$ the number density of Fe in phase $i$, and $f_{F e}(E)=32+f^{\prime}(E)+i f^{\prime \prime}(E)$ the effective number of electrons per Fe atom. The anomalous scattering factors, $f^{\prime}(E)$ and $f^{\prime \prime}(E)$, are apparent only near the particular atom's absorption edge. For this work, we work below the Fe and Ge K-edges, regions in energy where $f^{\prime}(E)$ is changing significantly but $f^{\prime \prime}(E)$ remains essentially constant.

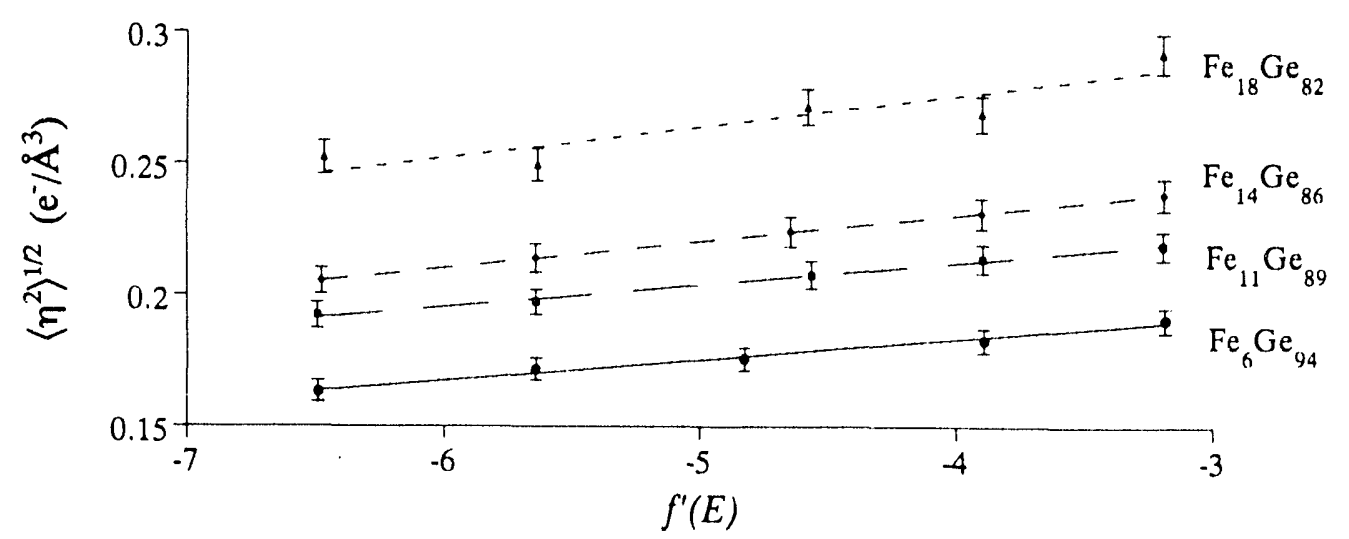

Figure 5. Plot of $\left\langle\eta^{2}\right\rangle$ versus $f^{\prime}(E)$ at the Fe K-edge for 4 different samples.

Since there is little if any change at the Ge K-edge, there is no resolvable change in $\left\langle\eta^{2}\right\rangle$ with energy at the $\mathrm{Ge}$ edge, so the number densities of $\mathrm{Ge}$ in both phases must be close to identical. At the Fe K-edge, however, there is a drastic change. The linear 
relation between $\left\langle\eta^{2}\right\rangle^{1 / 2}$ and $f^{\prime}(E)$ provides support for the validity of the model equation (figure 5). By writing out the volume fraction $c$ in terms of the atomic percent $x$, we have

$$
\left\langle\eta^{2}\right\rangle=\frac{x}{100-x}\left(1-\frac{x}{100-x} \frac{n_{G e}}{n_{F e}}\right) n_{F e} n_{G e}\left|f_{F e}(E)\right|^{2} .
$$

By determining $\left\langle\eta^{2}\right\rangle$ and with the knowledge of $\mathrm{x}$ and $f_{F e}(E)$, it is possible to determine $n_{\mathrm{Fe}}$ for a given $n_{\mathrm{Ge}}$ (usually $2-5 \%$ of the number density for crystalline $\mathrm{Ge}$ ). The atomic fraction $\mathrm{Fe}$ of the Fe-rich phase is then

$$
\frac{n_{F e}}{n_{F e}+n_{G e}}
$$

For number densities of 5\% less up to the crystalline value for $\mathrm{Ge}$, we have possible endpoints that vary from 33 to 37 atomic percent Fe for all samples studied. The number density of $\mathrm{Ge}$ in $\mathrm{c}-\mathrm{FeGe}_{2}$ is very similar to that of $\mathrm{c}-\mathrm{Ge}$, in agreement with the Ge edge result.

\section{CONCLUSIONS}

We have used oblique transmission ASAXS to better characterize the chemical homogeneity of a-Fe $\mathrm{Ge}_{\mathrm{x}} \mathrm{Ge}_{10 \mathrm{x}}$ films. A simple model that describes the strong anisotropy and agrees with the absolute scale measurements is a close-packing of oriented prolate ellipsoidal particles of a-FeGe 2 (a-Ge) in a background of a-Ge (a-FeGe $)_{2}$. These results are consistent with partial structure factors which have been determined using differential anomalous scattering and the total cylindrical distribution functions, which do not rely on a two-phase particle model and will be presented elsewhere. ${ }^{10}$

\section{ACKNOWLEDGEMENTS}

This research was performed at and supported by the Stanford Synchrotron Radiation Laboratory, which is funded by the Department of Energy through the Office of Basic Energy Science and the NIH, Biotechnology Resource Program, Division of Research Resources. The authors are indebted to P. Lecante, M. Rice, and R. Serimaa for assistance and suggestions concerning this work and to T. P. Russell for the use of his lupolen secondary standard.

\section{REFERENCES}

1M. Rice, S. Wakatsuki, and A. Bienenstock, J. Appl. Cryst. 24, 598 (1991); Mat. Res. Soc. Symp. 187, 53 (1990).

2R.D. Lorentz and A. Bienenstock, manuscript in preparation, 1993. R.D. Lorentz, PhD thesis, Stanford University, 1986.

3L.C. Wilson, PhD thesis, Stanford University, 1990.

4 ibid. See also M.J. Regan and M. Rice, SSRL Activity Report, 1993.

${ }^{5}$ G.S. Cargill, Phys. Rev. Lett. 28, 1372 (1972).

${ }^{6}$ T.P. Russell, J.S. Lin, S. Spooner, and G.D. Wignall, J. Appl. Cryst. 21, 629 (1988).

${ }^{7}$ M.J. Regan, PhD thesis, in preparation, Stanford University. M.J. Regan and A. Bienenstock, manuscript in preparation.

${ }^{8}$ A. Guinier and G. Fournet, Small-Angle Scattering of X-rays, (Wiley, New York, 1955). ${ }^{9}$ O. Glatter and O. Kratky, Small-Angle X-ray Scattering, (Academic Press, London, 1982).

10M.J. Regan and A. Bienenstock, to be presented at the IX International Conference on Small-Angle Scattering, Saclay, France, 1993. Proceedings published in J. de Phys. 

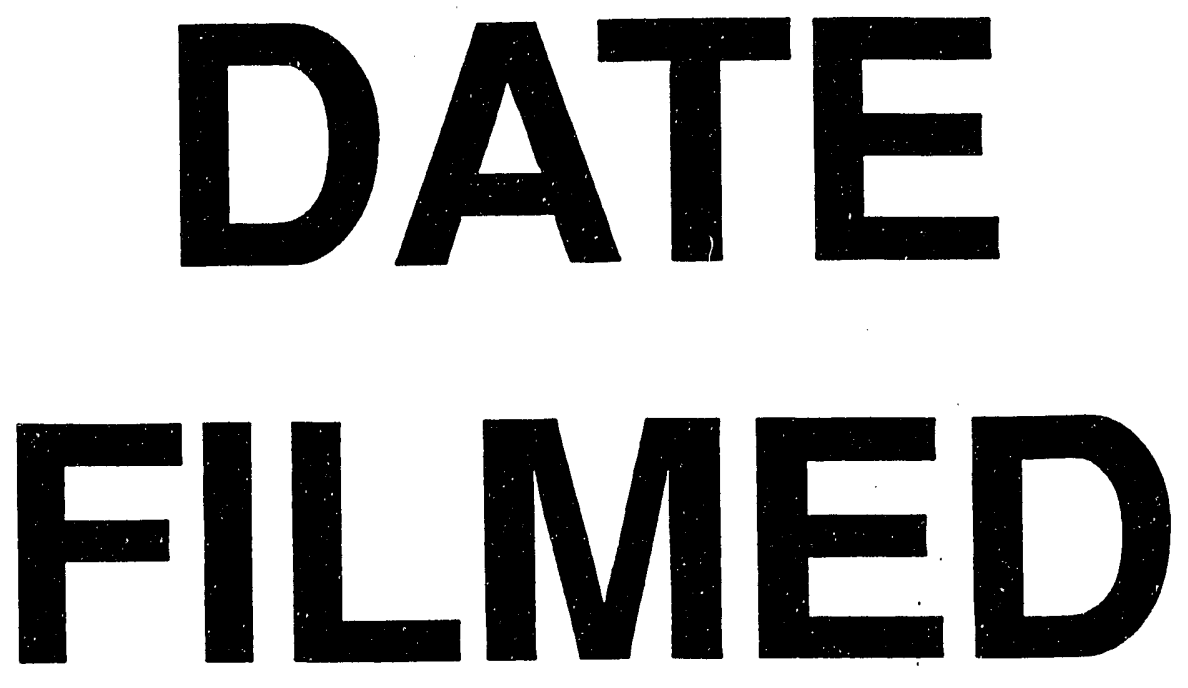

$8 / 23 / 93$
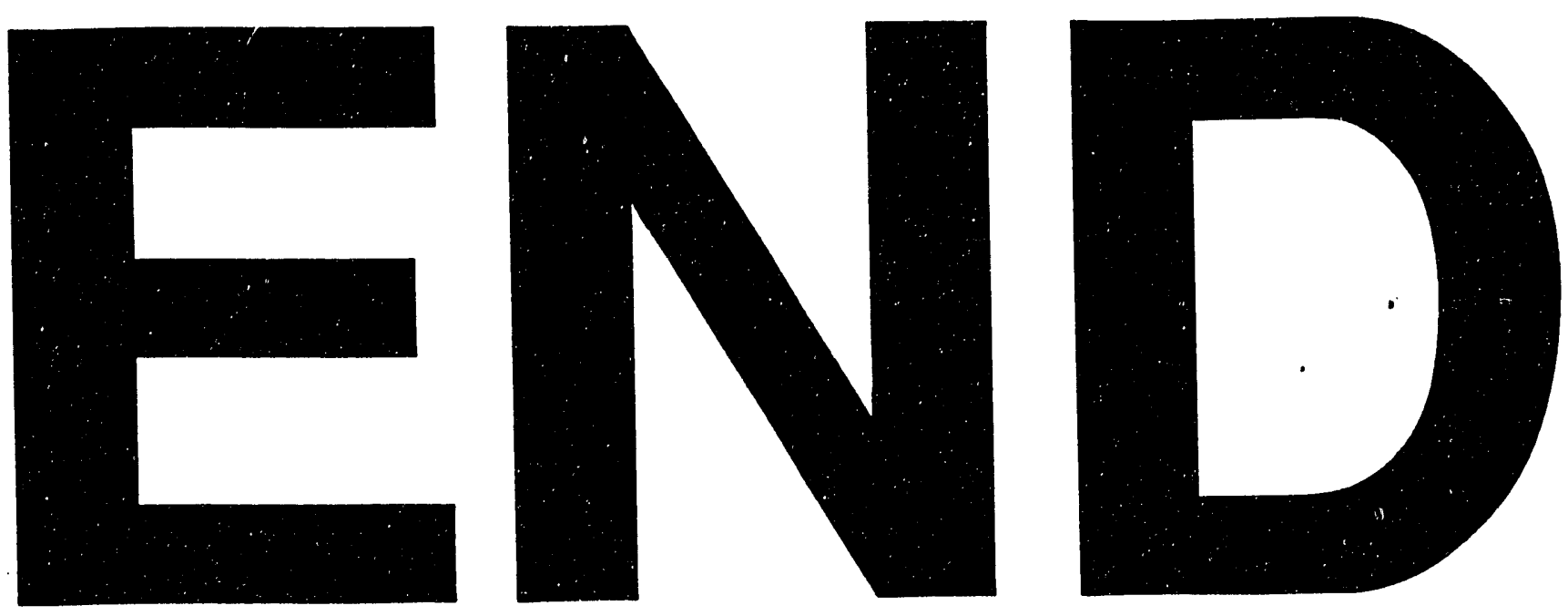
